\title{
Actualités dans la prise en charge du pied diabétique
}

\author{
Dr MARCELLE RORIVEa et Pr ANDRÉ J. SCHEEN ${ }^{a}$
}

Rev Med Suisse 2019; 15: 1448-52

\begin{abstract}
La prise en charge du pied diabétique doit être rapide face à toute plaie, si possible par une équipe multidisciplinaire. Le dépistage du pied à risque est la meilleure prévention. La neuropathie, sensitive, motrice et autonome, aboutit à des déformations, une perte de la protection algique et une sécheresse cutanée. Une artériopathie, également fréquente, hypothèque la cicatrisation des plaies, avec risque de surinfection et d'amputation. Les soins locaux et la mise en décharge sont essentiels, avec, si besoin, une antibiothérapie adaptée. La chirurgie curative mais aussi préventive, voire réparatrice, occupe une place grandissante. L'évaluation, le traitement et le suivi du pied diabétique répondent à des recommandations précises rédigées par I'IWGDF.
\end{abstract}

\section{News in the management of diabetic foot}

The management of diabetic foot requires a rapid intervention in front of any type of wound, if possible by a multidisciplinary team. The daily diabetic foot screening is the best prevention. Peripheral neuropathy leads to articular deformations, loss of pain alert and skin dryness. Arteriopathy, which is also frequent, retards cicatrisation of wounds, with a higher risk of infection and amputation. Meticulous local care and foot pressure off-loading are essential, with, if needed, appropriate antimicrobial therapy. Surgery, with curative, preventive or even restorative interventions, occupies an increasing place. The evaluation, treatment and follow-up of the diabetic foot should follow new guidelines established by the IWGDF.

\section{INTRODUCTION}

De nombreuses avancées ont été constatées ces dernières années dans le cadre de la prise en charge du pied diabétique. Elles sont, notamment, mises en application dans les «cliniques du pied», où est privilégiée une approche multidisciplinaire structurée (IWGDF : International Working Group on the Diabetic Foot). ${ }^{1}$ Cependant, il reste encore du travail quant à la prévention qui, in fine, représente, sans doute, le meilleur traitement du pied diabétique. ${ }^{2}$ La recherche de la neuropathie est la pierre angulaire de cette prévention. L'absence de sensibilité algique aura non seulement un rôle dans l'apparition de l'ulcération ou de la plaie, mais aussi dans le retard de cicatrisation en raison de la persistance de frottement ou compression non ressentis.

a Service de diabétologie, nutrition et maladies métaboliques, CHU Sart Tilman, 4000 Liège, Belgique mrorive@chuliege.be
Le présent article a pour but de décrire brièvement quelques grands principes de la prise en charge du pied diabétique, sur les plans préventif et thérapeutique.

\section{GÉNÉRALITÉS CONCERNANT LE PIED DIABÉTIQUE}

Le pied diabétique est défini comme étant toute plaie, infectée ou non, survenant au niveau du pied chez une personne diabétique présentant une neuropathie périphérique, sensitive et autonome, en présence de déformations et/ou des troubles circulatoires. ${ }^{3}$ On parle également de pied diabétique pour les déformations attribuées à l'arthropathie neurogène, appelée «pied de Charcot».

L'histoire clinique et la physiopathologie sont, généralement, assez stéréotypées. Certaines zones, irrégulières et proéminentes, subissent des pressions excessives et des microtraumatismes réguliers. Dans un premier temps, une hyperkératose réactionnelle se développe. Sans réaction adéquate du patient et/ou du soignant, elle va conduire à une ulcération (mal perforant). Celle-ci peut s'infecter plus ou moins rapidement et ne pas guérir rapidement, surtout s'il coexiste un tableau d'artériopathie périphérique. ${ }^{4}$

On distinguera alors l'ulcère aigu, qui doit conduire à une mise au point et une décharge adéquate, de l'ulcère chronique où la décharge de la zone lésée n'a pas été bien adaptée, retardant ainsi toute cicatrisation.

Plusieurs éléments cliniques doivent inciter à ce que les soignants de première ligne réfèrent le patient avec une problématique de pied diabétique à une équipe spécialisée de seconde ligne. ${ }^{5}$ Citons, notamment, une clinique d'insuffisance vasculaire, un contexte infectieux jugé défavorable (échec d'un traitement antibiotique dans une infection localisée, infection plus étendue, dont une cellulite, ou systémique, infection profonde avec contact osseux, mise en évidence de germes résistants), ou encore un contexte psychosocial défavorable. ${ }^{6}$

\section{PRÉVENTION DU PIED DIABÉTIQUE}

Si on veut avoir une attitude préventive efficace, il faut examiner le pied en pleine lumière, en passant sa main sur la plante des pieds, les zones interdigitales et l'extrémité des orteils (pulpes et articulations) à la recherche de déformations, de zones d'hyperkératose, d'ulcérations ou de mycoses. ${ }^{2,7}$ On observe le lit de l'ongle et la coupe. L'ongle sera coupé à l'horizontal et poncé à ses extrémités. La neuropathie et 
l'artériopathie membres inférieurs sont deux éléments cardinaux du pied diabétique et doivent être activement recherchées. Deux tests seront alors proposés en première intention. Le test au monofilament en 3 à 8 points afin de déterminer la perception ou non de ce dernier. L'absence de réponse témoigne d'une neuropathie sensitive avancée et doit conduire rapidement à des mesures préventives. ${ }^{8}$ Le second test est la palpation des pouls et le calcul de l'index de pression systolique (IPS) à la cheville, si l'on dispose d'un doppler portable. En cas de doute, une échographie artérielle des membres inférieurs sera alors demandée afin de préciser l'état vasculaire et distinguer les sténoses proximales des atteintes distales, ces dernières étant plus typiques du patient diabétique. ${ }^{9}$

Toute anomalie de l'un et/ou l'autre paramètre étudié doit conduire à des conseils appropriés. Citons, notamment, le port de chaussures sans couture, adaptées à ce type de problème, et de chaussettes en coton ou laine sans couture, ou encore la recommandation de ne pas marcher pieds nus que ce soit à l'extérieur, sur la plage par exemple, ou même à l'intérieur. Le patient doit également veiller à bien hydrater ses pieds à l'aide de crèmes hydratantes afin d'assouplir le revêtement cutané et réduire la formation classique des crevasses de talon. L'inspection des pieds avant et après le port des chaussures est conseillée. Un suivi de podologie sera organisé d'autant plus si le patient est porteur d'une neuropathie ou d'une artériopathie, a fortiori en cas d'atteinte mixte.

\section{CLASSIFICATION DES PLAIES DU PIED DIABÉTIQUE}

Plusieurs classifications ont été utilisées, essentiellement à des fins pronostiques (tableau 1). ${ }^{10}$ On utilisera la classification de Wagner pour son intérêt sur l'étude de la profondeur de la plaie et de l'atteinte vasculaire. ${ }^{11}$ Plus haut est le grade, plus élevé est le risque d'amputation. Malheureusement, elle ne donne pas d'information sur la présence d'une infection ou non, ni sur l'atteinte neurologique. La classification PEDIS a été proposée et adoptée comme alternative par l'International Working Group on the Diabetic Foot (IWGDF). Elle tient compte de la perfusion du pied (Perfusion), la dimension de la plaie (Extent), sa profondeur (Deep), l'infection (Infection) et la sensibilité conservée ou non (S). ${ }^{12}$ Plus descriptive, elle a une meilleure valeur prédictive. De plus, elle offre une échelle de communication commune entre les différents intervenants, médecins et paramédicaux. Elle permet, en effet, de décrire la plaie de façon très complète et de préciser son évolution au cours du temps, ce qui est capital si plusieurs intervenants successifs sont appelés à prendre en charge la même plaie. Une autre classification, utilisée également, est la classification de l'Université de Texas, qui associe l'aspect infecté ou non de la plaie, sa profondeur et/ou l'existence d'une atteinte vasculaire. ${ }^{13}$

Néanmoins, ces dernières classifications ne font pas toujours l'unanimité, raison pour laquelle, depuis quelques années, une nouvelle classification, appelée SINBAD (Site, Ischaemia, Neuropathy, bacterial Infection, Area, Depth), est proposée. ${ }^{14}$ Elle consiste à donner un point ou o en fonction de la question posée, ce qui simplifie la description avec un total maximum de 6 points. Cette classification, relativement simple à utiliser en pratique, est censée mieux informer le clinicien sur le temps de cicatrisation et le risque d'amputation selon la localisation de la plaie (tableau 1).

\section{TRAITEMENT DU PIED DIABÉTIQUE}

La plaie peut apparaître à différents endroits du pied. On reconnaît une plus grande prévalence pour les lésions d'orteils, soit pulpaires, soit au dos de l'orteil en griffe, soit encore en regard des têtes des métatarsiens, là où existent des zones d'hyperpression, mais toutes les autres localisations existent. Dans tous les cas, le bon pronostic dépend du fait que la plaie est diagnostiquée précocement, qu'elle est non infectée et qu'une décharge appropriée est rapidement mise en place.

On reconnaît comme facteur de non-cicatrisation : la neuropathie et l'absence de douleurs défensives, l'artériopathie, surtout distale, avec l'ischémie associée et l'altération du système immunitaire du patient diabétique. ${ }^{15}$ Ces facteurs de mauvais pronostic sont davantage présents lorsque le diabète évolue depuis plus de 10 ans, s'il est particulièrement mal équilibré au long cours, et s'il coexiste d'autres facteurs de risque comme un tabagisme, une hypertension artérielle et une dyslipidémie.

Rapidement, cette plaie devra être étudiée selon sa localisation, sa profondeur, la présence ou non d'un écoulement et d'un halo inflammatoire plus ou moins important. L'urgence dépendra de cet aspect, du degré d'inflammation et de sa diffusion vers l'ensemble du pied et/ou la présence de signes cliniques systémiques comme la fièvre, des frissons et des marqueurs biologiques inflammatoires comme une C-réactive

\begin{tabular}{l|l|l|l}
\hline & \multirow{2}{*}{ TABLEAU 1} & $\begin{array}{c}\text { Caractéristiques des systèmes } \\
\text { de classification pour les } \\
\text { ulcères du pied diabétique }\end{array}$ & \\
\cline { 5 - 6 } & & &
\end{tabular}

IWGDF : International Working Group on the Diabetic Foot.

\begin{tabular}{|c|c|c|}
\hline $\begin{array}{l}\text { Système de } \\
\text { classification }\end{array}$ & Points essentiels & Avantages/inconvénients \\
\hline Wagner ${ }^{11}$ & $\begin{array}{l}\text { Evalue la profondeur de } \\
\text { l'ulcère ainsi que la présence } \\
\text { de la gangrène et la perte de } \\
\text { perfusion à l'aide de six } \\
\text { grades (0 à 5) }\end{array}$ & $\begin{array}{l}\text { Bien établi } \\
\text { Ne traite pas complètement de } \\
\text { l'infection et de l'ischémie }\end{array}$ \\
\hline $\begin{array}{l}\text { Université du } \\
\text { Texas }^{12}\end{array}$ & $\begin{array}{l}\text { Evalue la profondeur de } \\
\text { l'ulcère, la présence de signes } \\
\text { d'une ischémie des membres } \\
\text { inférieurs à l'aide d'une } \\
\text { matrice de quatre grades } \\
\text { combinés à quatre stades }\end{array}$ & $\begin{array}{l}\text { Bien établi } \\
\text { Décrit mieux que Wagner la } \\
\text { présence d'une infection et } \\
\text { d'une ischémie et peut aider à } \\
\text { prévoir l'issue de l'ulcère du } \\
\text { pied diabétique }\end{array}$ \\
\hline PEDIS $^{13}$ & $\begin{array}{l}\text { Evalue la perfusion, l'étendue } \\
\text { (la taille), la profondeur } \\
\text { (perte tissulaire), l'infection } \\
\text { et la sensation (neuropathie) } \\
\text { au moyen de quatre grades } \\
\text { (1 à 4) }\end{array}$ & $\begin{array}{l}\text { Elaboré par l'IWGDF } \\
\text { Facile d'utilisation (définitions } \\
\text { claires, peu de catégories) } \\
\text { pour les praticiens qui } \\
\text { possèdent un faible niveau } \\
\text { d'expérience dans la prise en } \\
\text { charge du pied diabétique }\end{array}$ \\
\hline SINBAD ${ }^{14}$ & $\begin{array}{l}\text { Evalue l'emplacement, } \\
\text { l'ischémie, la neuropathie, } \\
\text { l'infection bactérienne, la } \\
\text { surface et la profondeur (par } \\
\text { une cotation binaire } 0 / 1 \text { ) }\end{array}$ & $\begin{array}{l}\text { Version simplifiée de } \\
\text { classification } \\
\text { Inclut notamment l'emplace- } \\
\text { ment de l'ulcère et les données } \\
\text { conduisant à penser qu'il } \\
\text { pourrait s'agir d'un déterminant } \\
\text { important de l'issue }\end{array}$ \\
\hline
\end{tabular}


protéine augmentée, une hyperleucocytose. La plaie sera nettoyée, débridée et des prélèvements bactériologiques seront effectués. Le cas échéant, le chirurgien donne également son avis quant à la prise en charge de la plaie (voir ci-après). Un drainage ou un débridement plus invasif sont souvent nécessaires pour des lésions délabrantes, larges et profondes.

Les examens complémentaires d'imagerie, par radiologie standard, résonance magnétique nucléaire ou tomodensitométrie, permettront de confirmer la présence ou non d'une ostéite, l'importance de l'atteinte des tissus mous (cellulite) et des tendons, la présence éventuelle de collections à drainer. L'échographie doppler artérielle renseignera sur l'étendue et la sévérité des lésions artérielles, et donc sur le risque de complications (dont la gangrène) ou de retard de cicatrisation. Un complément d'investigation sera alors proposé par le service de chirurgie vasculaire en vue d'une revascularisation.

Enfin, différents progrès technologiques ont été enregistrés au cours des dernières années qui aident à la prise en charge du pied diabétique. Citons, parmi d'autres, le développement de pansements actifs qui contribuent à créer un environnement optimal pour la cicatrisation, l'utilisation de la pression négative ${ }^{16}$ et la mise au point de différents modes de décharge selon la localisation de la plaie (voir ci-après), autant d'innovations qui permettent d'améliorer le pronostic.

\section{CHOIX DES ANTIBIOTIQUES FACE À UN PIED DIABÉTIQUE}

Devant un pied rouge et infecté, il est essentiel de collecter un maximum d'informations bactériologiques. ${ }^{17}$ Le prélèvement de matériel est le plus intéressant, qu'il soit issu d'un écoulement purulent, de débris locaux ou de morceaux de tissus (tendon, os). Des hémocultures seront réalisées également, a fortiori s'il existe un état fébrile.

En présence d'une lésion osseuse démontrée, que ce soit sur pied diabétique aigu ou chronique, la biopsie osseuse, après une fenêtre antibiotique de 10 jours, reste le «gold standard $\gg .{ }^{18}$ Dans ce type de complication, l'antibiothérapie sera adaptée aux résultats bactériologiques et proposée durant 6 à 12 semaines en fonction de l'évolution clinique. Dans les cas de plaies profondes ou accompagnées d'une cellulite, un traitement de 7 à 10 jours sera généralement proposé.

Les antibiotiques seront systématiquement choisis soit selon les recommandations internationales en l'absence de germes individualisés, soit sur la base de l'antibiogramme proposé par les analyses bactériologiques issues du laboratoire (tableau 2). Un suivi régulier doit être proposé et l'attitude thérapeutique doit être rapidement revue face à tout nouvel écoulement, un retard de cicatrisation, l'apparition d'un halo inflammatoire, une plaie luisante ou malodorante

\section{DÉCHARGE, ÉLÉMENT ESSENTIEL EN CAS DE PIED DIABÉTIQUE}

L'absence de décharge adéquate de la plaie conduira, inévitablement, à un retard de cicatrisation. On considère qu'une plaie prise en charge dans de bonnes conditions évoluera favorablement en 1 à 3 mois. En l'absence de décharge, on peut parfois attendre la cicatrisation plus d'une année, avec le risque ultime d'une amputation. ${ }^{19}$

\begin{tabular}{|c|c|c|c|}
\hline TABLEA & \multicolumn{2}{|c|}{$\begin{array}{l}\text { Recommandations pour une antibiothérapie empirique } \\
\text { dans les infections du pied diabétique }\end{array}$} & \\
\hline \multicolumn{4}{|c|}{ SASM : staphylocoque doré sensible à la méticilline ; SARM : staphylocoque doré résistant à la méticilline. } \\
\hline Sévérité de l'infection & Facteurs additionnels & Pathogène(s) habituel(s) & Antibiothérapie empirique potentielle \\
\hline \multirow{4}{*}{$\begin{array}{l}\text { Légère } \\
\text { (généralement traitement } \\
\text { oral) }\end{array}$} & Néant & $\begin{array}{l}\text { SASM, } \\
\text { Streptococcus spp }\end{array}$ & $\begin{array}{l}\text { Céphalosporine } 1^{\text {re }} \text { génération, ampicilline/sulbactam, } \\
\text { amoxycilline/clavulanate }\end{array}$ \\
\hline & $\begin{array}{l}\text { Allergie ou intolérance } \\
\text { aux bêtalactames }\end{array}$ & $\begin{array}{l}\text { SASM } \\
\text { Streptococcus spp }\end{array}$ & Clindamycine, lévofloxacine, moxifloxacine, doxycycline \\
\hline & Antibiothérapie récente & $\begin{array}{l}\text { SASM, } \\
\text { Streptococcus spp, germes Gram négatif }\end{array}$ & $\begin{array}{l}\text { Lévofloxacine, moxifloxacine, céphalosporine } 2^{\mathrm{e}} \text { ou } 3^{\mathrm{e}} \\
\text { génération }\end{array}$ \\
\hline & Haut risque de SARM & SARM & $\begin{array}{l}\text { Clindamycine, doxycycline, triméthoprime/ } \\
\text { sulfaméthoxazole }\end{array}$ \\
\hline \multirow{6}{*}{$\begin{array}{l}\text { Modérée } \\
\text { (traitement oral ou } \\
\text { initiation parentérale puis } \\
\text { relais oral) ou sévère } \\
\text { (traitement parentéral) } \\
\text { Antibiothérapie récente }\end{array}$} & Néant & $\begin{array}{l}\text { SASM, } \\
\text { Streptococcus } \\
\text { spp } \pm \text { germes } \\
\text { Gram négatif }\end{array}$ & Céphalosporine $2^{\mathrm{e}}$ ou $3^{\mathrm{e}}$ génération \pm aminoglycoside \\
\hline & Antibiothérapie récente & $\begin{array}{l}\text { SASM, Streptococcus spp } \pm \text { germes Gram } \\
\text { négatif }\end{array}$ & $\begin{array}{l}\text { Céphalosporine } 3^{e} \text { génération } \pm \text { aminoglycoside, } \\
\text { pipéracilline/tazobactam }\end{array}$ \\
\hline & Ulcère avec macération & Germes Gram négatif, incluant Pseudomonas & Pipéracilline/tazobactam, imipénème, méropéneme \\
\hline & $\begin{array}{l}\text { Ischémie/nécrose/ } \\
\text { formation de gaz }\end{array}$ & $\begin{array}{l}\text { SASM, Streptococcus spp. } \pm \text { germes Gram } \\
\text { négatif } \pm \text { germes anaérobies }\end{array}$ & $\begin{array}{l}\text { Pipéracilline/tazobactam, céphalosporine } 2^{\mathrm{e}} \text { ou } 3^{\mathrm{e}} \\
\text { génération ou céfépime + clindamycine ou métronidazole }\end{array}$ \\
\hline & Haut risque de SARM & $\begin{array}{l}\text { SARM, Streptococcus spp. } \pm \text { germes Gram } \\
\text { négatif }\end{array}$ & $\begin{array}{l}\text { Vancomycine ou teicoplanine + céphalosporine } 3^{\text {e }} \\
\text { génération, céfépime, pipéracilline/tazobactam }\end{array}$ \\
\hline & $\begin{array}{l}\text { Risque de germes Gram } \\
\text { négatif résistants }\end{array}$ & $\begin{array}{l}\text { Germes produisant des bêtalactamases à large } \\
\text { spectre, germes Gram négatif multirésistants }\end{array}$ & $\begin{array}{l}\text { Pipéracilline/tazobactam + aminoglycoside, imipénème, } \\
\text { méropéneme }\end{array}$ \\
\hline
\end{tabular}

(Adapté de réf.17). 
Selon Armstrong et coll. ${ }^{19}$ le total aircast ou plâtre reste la meilleure solution. On observe un cicatrisation plus rapide. Malheureusement, selon la localisation des lésions, en particulier si elles sont plantaires ou talonnières, les soins infirmiers sont alors difficiles à réaliser. Dans ce cas, des chaussures ou bottes de décharge seront proposées. La classification SINBAD, déjà décrite, est assez aisée à utiliser et donne une idée du pronostic et de la durée probable du traitement. ${ }^{14}$ On note un vieillissement de la population et un tel score nous apporte une information utile en ce qui concerne le temps supposé pour obtenir une cicatrisation, ce qui doit nous permettre de réfléchir sur le choix de la prise en charge.

Cette réflexion est d'autant plus nécessaire si nous sommes confrontés à une plaie profonde chronique, ne cicatrisant pas depuis plus de 3 mois, chez un patient gériatrique. La qualité de vie du patient doit rester à l'esprit dans le choix de la prise en charge, en tenant compte des aptitudes locomotrices et de la possibilité de décharge, sans risque de chute, sans oublier le pronostic vital.

\section{PRISE EN CHARGE CHIRURGICALE DU PIED DIABÉTIQUE}

L’objectif des «cliniques du pied», grâce à leur multidisciplinarité, est de permettre au patient diabétique d'accéder à une prise en charge rapide et efficace, si possible salvatrice même en cas de lésions avancées. Le recours au chirurgien peut s'avérer nécessaire dans différentes situations, en prévention secondaire ou, parfois, même en prévention primaire. ${ }^{20}$ Classiquement, la chirurgie face à un pied diabétique peut être proposée en prévention secondaire, par exemple pour procéder à une amputation suite à une ostéomyélite ne répondant pas au traitement antibiotique classique. Il peut s'agir, le plus souvent, d'une amputation mineure (ostéotomie) telle celle d'un orteil, mais il est parfois nécessaire de recourir à une amputation majeure telle celle de l'avant-pied, voire même de la jambe. La prise en charge multidisciplinaire a pour intérêt, lorsque l'amputation est inévitable, d'en limiter l'étendue et la rendre la plus ciblée possible, de façon à garantir ultérieurement un chaussage adéquat.

Cependant, il est également fait mention, maintenant, d'une chirurgie en prévention primaire, c'est-à-dire avant l'apparition de toute plaie. Elle consiste, avant tout, à corriger les déformations douloureuses ou à haut risque de favoriser une plaie ultérieurement. Elle peut également être considérée lors d'une asymétrie majeure entre les deux pieds avec des problèmes de chaussage tels que l'hallux valgus, le pied de Charcot. Ce type de chirurgie peut également être proposé en prévention secondaire afin d'éviter la récidive tel l'ulcère pulpaire de l'hallux rigidus ou d'un orteil en griffe.

Le recours au chirurgien ne se limite cependant pas à l'amputation. Le chirurgien vasculaire joue un rôle essentiel en cas d'indication de la revascularisation du membre en présence d'une plaie ischémique ou neuro-ischémique. Les techniques de revascularisation ont fait des progrès importants ces dernières années, avec réalisation de pontages sur des artères de calibre de plus en plus petit, y compris au niveau de la cheville. Par ailleurs, comme déjà mentionné, le débridement chirurgical est également un acte essentiel en présence d'un ulcère compliqué de cellulite, voire d'ostéite.

Malheureusement, le risque chirurgical n'est jamais faible quand on sait que le patient diabétique a un risque d'infection ou de complication qui est 3 à 6 fois supérieur à celui d'un sujet non diabétique. La décision d'intervenir doit toujours être discutée en présence de la famille et en tenant compte de l'âge, de la mobilité, des problèmes sociaux et de l'état psychologique du patient.

\section{CONCLUSION}

La prise en charge du pied diabétique ne peut plus se concevoir par un seul prestataire. Un encadrement multidisciplinaire est indispensable pour améliorer la qualité de la prise en charge et réduire le temps de cicatrisation. En cas de lésion, la décharge est indispensable. Elle doit être adaptée à chaque patient selon la présence d'une ou plusieurs plaies, leur localisation et la mobilité du patient. Il faut alors envisager une aide complémentaire par béquilles, chaise roulante, canne.

La prévention reste le meilleur traitement et il faut encourager le dépistage de la neuropathie sensitive annuellement et la surveillance des déformations. Le podologue a une place essentielle dans cette approche préventive. Des ateliers éducatifs, comprenant le soin des pieds, l'évaluation du risque et le chaussage adéquat, sont des initiatives, simples mais essentielles, qui permettront certainement de réduire encore le nombre d'amputations chez les personnes diabétiques.

Conflit d'intérêts : Les auteurs n'ont déclaré aucun conflit d'intérêts en relation avec cet article.

\section{IMPLICATIONS PRATIQUES}

- Surveillance régulière de l'architecture et des zones d'appui chez un patient diabétique depuis plus de 10 ans

- Dépistage de la neuropathie sensitive par le test du monofilament

- Dépistage de l'artériopathie oblitérante par le calcul de IPS (index Cheville-Bras)

- Décharge immédiate en présence de toute lésion au pied (chaussure orthopédique, botte amovible ou plâtrée)

- Antibiothérapie empirique puis ciblée dès réception des résultats bactériologiques

- Toujours envisager la place du chirurgien quel que soit le type de plaie 
1 ** International Working Group on the Diabetic Foot. IWGDF Guidelines on the prevention and treatment of diabetic foot disease. 2019. https://iwgdfguidelines.org (dernier accès 15 juin 2019).

2 * Schaper NC, Van Netten JJ, Apelqvist $J$, et al; International Working Group on the Diabetic Foot (IWGDF). Prevention and management of foot problems in diabetes : a summary guidance for daily practice 2015, based on the IWGDF

guidance documents. Diabetes Res Clin Pract 2017;124:84-92.

3 Diabetes Canada Clinical Practice Guidelines Expert Committee, Embil JM, Albalawi Z, Bowering K, et al. Foot care. Can J Diabetes 2018;42 (Suppl. 1):S222-7. 4 Malgrange D. Physiopathologie du pied diabétique. Rev Med Int 2008;29 (Suppl.2):S231-7.

5 Van Acker K, Vandeleene B, Leemrijse T. Prise en charge du pied diabétique dans un centre spécialisé. In : Prise en charge du pied diabétique. Hoboken,
Belgique : Albe De Coker, 2008; p. 278. 6 Orioli L, Vandeleene B. La prise encharge du pied diabétique : de la nécessité d'une équipe pluridisciplinaire. Louvain Médical 2017;136:187-94. 7 Martini J. Le pied diabétique : dépistage et prévention. Rev Med Int 2008;29(Suppl.2):S260-3.

8 Richard JL, Reilhes L, Buvry S, et al. Screening patients at risk for diabetic foot ulcérations: a comparaison between measurement of vibration perception threshold and 10 gr monofilament test. Int Wound J 2014;11:147-51.

9 Wu S, Driver VR, Wrobel JS, Armstrong DG. Foot ulcers in the diabetic patient prevention and treatment. Vasc Health Risk Manag 2007;3:65-76.

10 Expert working group; Best practice guidelines: wound management in diabetic foot ulcer. Wounds International 2013. www.Wounds international.com (dernier accès 15 juin 2019).

11 Wagner FW. The dysvascular foot : a system of diagnosis and treatment. Foot Ankle 1981;2:64-122.

12 * Lipsky BA, Berendt AR, Cornia PB, et al; Infectious Diseases Society of America. 2012 Infectious Diseases Society of America clinical practice guideline forthe diagnosis and treatment of diabetic foot infections. Clin Infect Dis2012;54:e132-73. 13 Armstrong DG, Lavery LA, Harkless LB. Validation of a diabetic wound classification system : the contribution of depth, infection and vascular disease to the risk of amputation. Diabetes Care 1998;21:855-9.

14 * Ince P, Abbas ZG, Lutale JK, et al. Use of the SINBAD classification system and score in comparing outcome of foot ulcer management on three continents. Diabetes Care 2008;31:964-7.

15 Cavanagh PR, Lipsky BA, Bradbury AW, Botek G. The treatment for diabetic foot ulcers. Lancet 2005;366:1725-35.

16 Wynn M, Freeman S. The efficacy of negative pressure wound therapy for diabetic foot ulcers: A systematised review. J Tissue Viability 2019; epub ahead of print.

17 Kwon KT, Amstrong DG. Microbiology and antimicrobial therapy for diabetic foot infections. Infect Chemother 2018;50:11-20.

18 Senneville E, Lombart A, Beltrand E, et al. Outcome of diabetic foot osteomyelitis treated nonsurgically: a retrospective cohort study. Diabetes Care 2008;31:63742.

19 Armstrong DG, Nguyen HC, Lavery LA, et al. Offloading the diabetic foot wound: a randomized clinical trial. Diabetes Care 2001;24:1019-22.

20 Caputo WJ. Surgical management of the diabetic foot. Wounds 2008;20:74-83.

* à lire

** à lire absolument 